\title{
DNA-based control of oak wood geographic origin in the context of the cooperage industry
}

\author{
Marie-France DeguillouX ${ }^{\mathrm{a}, \mathrm{b}}$, Marie-Hélène PEMONGE ${ }^{\mathrm{a}}$, Rémy J. PeTiT ${ }^{\mathrm{b} *}$ \\ a Institut National de la Recherche Agronomique, Unité de Recherches Forestières, Équipe de Génétique des Arbres Forestiers, \\ 69 Route d'Arcachon, 33612 Cestas Cedex, France \\ ${ }^{\text {b }}$ Centre Technique du Bois et de l'Ameublement, 10 avenue de St-Mandé, 75012 Paris, France
}

(Received 18 September 2002; accepted 20 May 2003)

\begin{abstract}
The recent growth of the French barrel industry, leading to increased importations of oak wood and a general lack of wood origin guarantee, has resulted in the demand for a reliable technique permitting to control the provenance of oak wood. In this study we propose to adapt wood traceability technique using chloroplast DNA markers to this industrial context. The retrieval of DNA and haplotype determination has been tested on different types of wood samples that can be collected in cooperage firms, and a clear effect of wood treatment on DNA degradation has been observed. Despite the poor quantity and quality of DNA retrieved from staves, haplotypes could be determined on a large proportion of the samples, permitting to check the genetic conformity of woods with announced provenances. In several cases, our study proved the existence of unlabeled oak woods originating from eastern Europe and the incorrect use of the names of famous French forests.
\end{abstract}

chloroplast DNA / diversity / haplotype / Quercus / traceability

Résumé - Contrôle de l'origine géographique des bois de chêne utilisés en tonnellerie. L'importation croissante de bois de chêne et le manque de garantie sur son origine, liés à l'expansion récente de la tonnellerie française, rendent nécessaire la mise au point d'une technique fiable permettant de contrôler l'origine des bois de chêne. Dans cette étude, nous avons cherché à adapter, pour ce secteur industriel, les méthodes moléculaires de traçabilité des bois de chêne utilisant les marqueurs chloroplastiques. La qualité et la quantité de l'ADN extrait et la caractérisation des haplotypes ont alors été évalués sur les différents types d'échantillons de bois rencontrés dans les tonnelleries. Nous avons pu démontrer un effet net des différents traitements du bois sur la dégradation de l'ADN. Cependant, malgré la faible qualité et quantité d'ADN extrait des bois de merrains, les haplotypes ont pu être déterminés sur une large proportion des échantillons, permettant de tester la conformité des bois avec l'origine annoncée. Dans plusieurs cas, nous avons pu mettre en évidence l'existence de bois mal identifiés, provenant de l'est de l'Europe, ainsi que l'utilisation abusive des noms des provenances françaises renommées.

ADN chloroplastique / diversité / haplotype / Quercus / traçabilité

\section{INTRODUCTION}

Decades ago winemakers discovered that certain wines benefited from storage in oak barrels [2, 18]. The barrel essentially does two things: it allows a very slow introduction of oxygen into the wine, and it imparts the character of the wood into the wine by leaching of extractives. In this way, wine goes through subtle chemical changes, resulting in greater complexity and a softening of the harsh tannins and flavours present at the end of fermentation. Only European pedunculate and sessile oaks (respectively Quercus robur L. and Quercus petraea (Matt.) Liebl.), as well as American white oaks (especially Quercus alba L.), satisfy the requirements of porosity, strength and flavour to be imparted to the finished wine. Recent growth, investment and modernization in the wine and spirit industry have created a great demand for oak logs or staves (i.e. the narrow strips of wood used to make up the barrel).

The cooperage market is currently the most profitable market for oak wood in France, with a growth rate of $15 \%$ per year and up to $200000 \mathrm{~m}^{3}$ of oak wood removed per year for a total of 400000 barrels produced (data communicated by the Fédération Française de Tonnellerie). Much of the wood used by the French barrel industry still originates from France. Oaks (especially $Q$. robur and $Q$. petraea) are indeed the most abundant tree species in the French forests, which cover some $27 \%$ of the land, and the quality of French oak is a renowned standard. However, the recent growth of the barrel industry has forced French coopers to import significant amounts of oak wood, either from eastern European countries (mostly $Q$. robur) or from the United States (for Quercus alba). Consequently,

\footnotetext{
*Corresponding author: petit@ pierroton.inra.fr
} 
the effect of the geographic origin of oak wood on wines is the subject of many discussions and experimentations throughout the world $[10,13,14]$, along with the influence of barrel making process itself on wine (i.e. open-air drying of staves, wood toasting) $[3,6,9,10,15,17,19,20]$. Another consequence of the diversification of oak wood origins is the need for clear provenance identification.

The recent trade of oak wood between eastern European countries, United States and France has developed because of an increased demand from the cooperage industry and because of differences in price. In this context, the origin of oak wood is difficult to guarantee, especially within Europe, since the same species are found throughout much of the continent ( $Q$. petraea and especially $Q$. robur). In particular, the lack of clear identification of provenances during transactions, coupled with the variable but often high number of intermediaries between the oak forest and the cooper, are often reported. This results in a demand from the cooper industry for a reliable technique permitting to control the provenance of oak wood. The ultimate rationale for an improved "traceability" is the respect of prices and should eventually benefit to foresters producing high quality oak wood in sustainably managed forests.

Previous research indicates that maternally inherited DNA markers located in the oak chloroplast genome should be able to meet some of the expectations for traceability in this industry, provided DNA could be retrieved from dry wood. Methods comparing strontium isotopes ratios have also been proposed to identify logs of spruce or fir from different sites [8]. Because these approaches, although encouraging, are limited by the fact that such markers can differ between individuals from the same population or even between different tissues from the same individual [11], we have started to explore the possibilities of genetic markers.

We recently developed a wood traceability technique using chloroplast DNA markers in European oaks [5]. In particular, we first demonstrated the possibility to recover and analyse DNA from dry wood $[4,7]$. The next step consisted in a transposition of the characterisation of chloroplast variants (i.e. haplotype) from DNA isolated from fresh samples onto DNA isolated using dry wood samples; such variants can be used to differentiate oak wood lots originating from western versus eastern Europe [5]. Chloroplast (cp) DNA lineages have been previously identified and mapped in European oaks [16]. For the most part, their distribution was established during postglacial expansion of oaks from distinct refugia, after the last ice age (approximately 18000 years before Present). Different recolonisation routes involving different European refugia resulted in a clear geographical structure of cpDNA haplotypes. This strong structure, uncovered by mapping the cpDNA genetic structure at over 2600 localities, can be used for traceability purposes. In fact, oak stands often possess only one haplotype, so that conformity tests of oak products with their announced origin are considerably facilitated.

Here this method is adapted to the case of the French cooperage industry, to help coopers check the conformity of oak wood lots. The retrieval of DNA and haplotype determination has been tested on wood samples collected in several cooperage firms. We tested the complete traceability technique on all types of wood samples that might be used for provenance control in this industry. Tests were conducted on green staves seasoned less than one year outdoors, seasoned staves entering in the manufacture chain (after two years of open-air drying), and staves collected from finished barrels (i.e. staves that have followed the entire manufacture chain, including the toasting step). The feasibility of DNA analysis on those different types of samples is compared and a global traceability process adapted to the context of cooperage is proposed.

\section{MATERIALS AND METHODS}

\subsection{Wood material}

A total of 131 oak wood samples $(Q$. robur and $Q$. petraea $)$ were collected in ten different French cooperage makers, corresponding to three steps of barrels making (green, dry or barrel staves, Tab. I). In five firms, green and/or dry staves were collected in open-air wood stocks (firms A-B-C-D-E), whereas in five other firms dry staves were collected at the beginning of the barrel making process (firms FG-H-I-J). Note however that the samples are not necessarily representative of wood diversity in those firms. In total, tests were conducted on 56 green staves and nine logs seasoned less than one year outside, 36 seasoned staves entering in the manufacture chain (after two years of open-air drying) and 30 staves collected from a single finished barrel (Tab. I). The assumed provenances of all wood samples were provided by the cooperages before analysis, provenance information ranging from the European region to the stand. Among the 56 green staves, 17 had been prepared from the nine logs, in order to check the conformity of the haplotypes revealed when different parts of a log are used for analysis. These were labelled "Green a-i" (for the staves) and "GreenLog a-i" (for the logs). The barrel investigated was prepared specifically for this study. It was made up of staves originating from various regions in France and from different countries (Tab. I). The information about the staves used to make the barrel was kept secret until the analysis was completed, in order to check the validity of the procedure.

\subsection{DNA isolation and amplification}

A little piece of wood was sawn from each stave and used for DNA isolation. All wood DNA isolation procedures were carried out under sterile conditions in separate dedicated rooms, as described in Deguilloux et al. [4]. The procedure first included cleaning with diluted bleach and suppression of surface tissues of wood fragments. Then, less than $100 \mathrm{mg}$ of wood shavings was obtained with a scalpel from internal parts of samples, or parts that were not directly toasted in the case of staves obtained from the barrel (at each extremity of the staves). Those shavings were then ground into a fine powder using a Retsch-mill apparatus (Fischer-Bioblock) and used for genomic DNA isolation with the DNeasy Plant minikit (Qiagen). During each isolation manipulation, several negative controls (treated in the same way than the normal samples except that no sample sawdust was added) were used in order to check for potential contamination.

The amplification of 11 different cpDNA fragments, allowing the haplotype characterisation of wood samples (Tab. II), was performed according to Deguilloux et al. [5]. Three different sets of fragments were used to characterize chloroplast variants: the combination I (involving three longer fragments easier to score: $\mathrm{d} 1 \mathrm{t} 1, \mathrm{~d} 7 \mathrm{t} 7$ and $\mathrm{t} 4 \mathrm{f} 4$ ) was used for haplotype determination on green wood samples, the combination II (involving three shorter fragments easier to amplify on degraded DNA: dt12b, dt72 and tf42) was developed for drier wood; and finally the combination III (involving five even shorter fragments: dt14, $\mu \mathrm{dt} 1$, dt73, dt74 and tf42) was used for staves 
Table I. Type, provenance, haplotype and conformity with announced origin of analysed staves.

\begin{tabular}{|c|c|c|c|c|c|c|}
\hline Sample & Type of stave & Cooperage & $\begin{array}{l}\text { Announced } \\
\text { provenance }\end{array}$ & $\begin{array}{c}\text { Provenance } \\
\text { information type }\end{array}$ & Haplotype & $\begin{array}{c}\text { Conformity with } \\
\text { provenance haplotypes }\end{array}$ \\
\hline Green 1 & green stave & A & Vosges & region & $\mathrm{b}$ & yes \\
\hline Green 2 & green stave & A & Vosges & region & $c-d$ & yes \\
\hline Green 3 & green stave & A & Vosges & region & $\mathrm{e}$ & yes \\
\hline Green 4 & green stave & A & Vosges & region & $\mathrm{c}$ & yes \\
\hline Green 5 & green stave & A & Vosges & region & $\mathrm{d}$ & yes \\
\hline Green 6 & green stave & A & Vosges & region & $\mathrm{b}$ & yes \\
\hline Green 7 & green stave & A & Vosges & region & $\mathrm{b}$ & yes \\
\hline Green 8 & green stave & A & Vosges & region & $\mathrm{b}$ & yes \\
\hline Green 9 & green stave & A & Vosges & region & $\mathrm{b}$ & yes \\
\hline Green 10 & green stave & A & Nevers & region & $\mathrm{c}$ & yes \\
\hline Green 11 & green stave & A & Nevers & region & $\mathrm{b}$ & yes \\
\hline Green 12 & green stave & A & Nevers & region & $\mathrm{b}$ & yes \\
\hline Green 13 & green stave & A & Vosges & region & $\mathrm{b}$ & yes \\
\hline Green 14 & green stave & A & Vosges & region & $\mathrm{b}$ & yes \\
\hline Green 15 & green stave & A & Vosges & region & $\mathrm{b}$ & yes \\
\hline Green 16 & green stave & A & Vosges & region & - & - \\
\hline Green 17 & green stave & A & Vosges & region & $\mathrm{b}$ & yes \\
\hline Green 18 & green stave & A & Vosges & region & $\mathrm{b}$ & yes \\
\hline Green 19 & green stave & A & Nevers & sub-region & $\mathrm{b}$ & yes \\
\hline Green 20 & green stave & A & Nevers & sub-region & $\mathrm{b}$ & yes \\
\hline Green 21 & green stave & A & Nevers & sub-region & $\mathrm{c}$ & yes \\
\hline Green 22 & green stave & A & Tronçais & stand & $\mathrm{b}$ & no \\
\hline Green 23 & green stave & A & Tronçais & stand & $\mathrm{b}$ & no \\
\hline Green 24 & green stave & A & Tronçais & stand & $\mathrm{c}$ & yes \\
\hline Green 25 & green stave & A & Vosges & region & $\mathrm{b}$ & yes \\
\hline Green 26 & green stave & A & Vosges & region & $\mathrm{b}$ & yes \\
\hline Green 27 & green stave & A & Vosges & region & $\mathrm{b}$ & yes \\
\hline Green 28 & green stave & A & Vosges & region & - & - \\
\hline Green 29 & green stave & A & Vosges & region & $\mathrm{b}$ & yes \\
\hline Green 30 & green stave & A & Vosges & region & - & - \\
\hline Green 31 & green stave & A & Vosges & region & $\mathrm{b}$ & yes \\
\hline Green 32 & green stave & A & Centre & region & $\mathrm{a}$ & - \\
\hline Green 33 & green stave & A & Centre & region & $\mathrm{b}$ & yes \\
\hline Green 34 & green stave & A & Vosges & region & $\mathrm{b}$ & yes \\
\hline Green 35 & green stave & A & Vosges & region & $\mathrm{b}$ & yes \\
\hline Green 36 & green stave & A & Vosges & region & $\mathrm{b}$ & yes \\
\hline Green 37 & green stave & A & Vosges & region & $\mathrm{b}$ & yes \\
\hline Green 38 & green stave & A & Centre & region & $\mathrm{b}$ & yes \\
\hline Green 39 & green stave & B & Jupilles & stand & $\mathrm{b}$ & no \\
\hline Green Log a & green log & B & Jupilles & stand & $\mathrm{b}$ & no \\
\hline Green a & green stave & B & Jupilles & stand & $\mathrm{b}$ & no \\
\hline Green a' & green stave & B & Jupilles & stand & $\mathrm{b}$ & no \\
\hline Green Log b & green log & B & Haguenau & stand & $\mathrm{b}$ & yes \\
\hline Green b & green stave & B & Haguenau & stand & $\mathrm{b}$ & yes \\
\hline Green b' & green stave & B & Haguenau & stand & $\mathrm{b}$ & yes \\
\hline Green Log c & green log & B & Tronçais & stand & $\mathrm{b}$ & no \\
\hline Green c & green stave & B & Tronçais & stand & $\mathrm{b}$ & no \\
\hline Green c' & green stave & B & Tronçais & stand & $\mathrm{b}$ & no \\
\hline Green Log d & green log & B & Oison & stand & $\mathrm{f}$ & no \\
\hline Green d & green stave & B & Oison & stand & $\mathrm{f}$ & no \\
\hline Green d' & green stave & B & Oison & stand & $\mathrm{f}$ & no \\
\hline Green Log e & green log & B & Haute Saône & region & $\mathrm{b}$ & yes \\
\hline Green e & green stave & $\mathrm{B}$ & Haute Saône & region & $\mathrm{b}$ & yes \\
\hline Green e' & green stave & B & Haute Saône & region & $\mathrm{b}$ & yes \\
\hline Green Log f & green log & $\mathrm{B}$ & Jupilles & stand & $\mathrm{b}$ & no \\
\hline Green $\mathrm{f}$ & green stave & B & Jupilles & stand & $\mathrm{b}$ & no \\
\hline Green $\mathrm{f}^{\prime}$ & green stave & $\mathrm{B}$ & Jupilles & stand & $\mathrm{b}$ & no \\
\hline Green Log g & green log & $\mathrm{B}$ & Lunéville & stand & $\mathrm{b}$ & yes \\
\hline Green $g$ & green stave & $\mathrm{B}$ & Lunéville & stand & $\mathrm{b}$ & yes \\
\hline Green g' & green stave & $\mathrm{B}$ & Lunéville & stand & $\mathrm{b}$ & yes \\
\hline Green Log h & green log & $\mathrm{B}$ & Nièvre & region & $\mathrm{b}$ & yes \\
\hline Green $\mathrm{h}$ & green stave & $\mathrm{B}$ & Nièvre & region & $\mathrm{b}$ & yes \\
\hline Green $h^{\prime}$ & green stave & $\mathrm{B}$ & Nièvre & region & $\mathrm{b}$ & yes \\
\hline Green Log i & green log & B & Fontainebleau & stand & $\mathrm{c}$ & yes \\
\hline
\end{tabular}


Table I. Continued.

\begin{tabular}{|c|c|c|c|c|c|c|}
\hline Sample & Type of stave & Cooperage & $\begin{array}{l}\text { Announced } \\
\text { provenance }\end{array}$ & $\begin{array}{c}\text { Provenance } \\
\text { information type }\end{array}$ & Haplotype & $\begin{array}{c}\text { Conformity with } \\
\text { provenance haplotypes }\end{array}$ \\
\hline Green i & green stave & $\mathrm{B}$ & Fontainebleau & stand & - & - \\
\hline Dry 1 & dry stave & $\mathrm{E}$ & Tronçais & stand & $\mathrm{c}$ & yes \\
\hline Dry 2 & dry stave & $\mathrm{E}$ & $?$ & & $\mathrm{~d}$ & - \\
\hline Dry 3 & dry stave & $\mathrm{E}$ & Vosges & region & $\mathrm{a}$ & yes \\
\hline Dry 4 & dry stave & $\mathrm{C}$ & Pyrenees & region & $\mathrm{c}$ & yes \\
\hline Dry 5 & dry stave & $\mathrm{D}$ & Alsace & region & $\mathrm{h}$ & no \\
\hline Dry 6 & dry stave & $\mathrm{C}$ & Nevers & region & $\mathrm{c}$ & yes \\
\hline Dry 7 & dry stave & $\mathrm{L}$ & Poland & country & $\mathrm{h}$ & yes \\
\hline Dry 8 & dry stave & $\mathrm{C}$ & eastern Europe & European region & $\mathrm{h}$ & yes \\
\hline Dry 9 & dry stave & $\mathrm{D}$ & Vosges & region & $\mathrm{b}$ & yes \\
\hline Dry 10 & dry stave & $\mathrm{D}$ & Nièvre & region & $\mathrm{b}$ & yes \\
\hline Dry 11 & dry stave & $\mathrm{C}$ & Vosges & region & $\mathrm{a}$ & yes \\
\hline Dry 12 & dry stave & $\mathrm{C}$ & $?$ & & $\mathrm{a}$ & - \\
\hline Dry 13 & dry stave & $\mathrm{C}$ & Pyrenees & region & $\mathrm{c}$ & yes \\
\hline Dry 14 & dry stave & $\mathrm{H}$ & Vosges & region & $\mathrm{c}$ & yes \\
\hline Dry 15 & dry stave & $\mathrm{H}$ & Vosges & region & $\mathrm{c}$ & yes \\
\hline Dry 16 & dry stave & $\mathrm{H}$ & Vosges & region & $\mathrm{c}$ & yes \\
\hline Dry 17 & dry stave & $\mathrm{H}$ & Vosges & region & $\mathrm{c}$ & yes \\
\hline Dry 18 & dry stave & D & Allier & region & $\mathrm{c}$ & yes \\
\hline Dry 19 & dry stave & $\mathrm{D}$ & Allier & region & c-e & yes \\
\hline Dry 20 & dry stave & $\mathrm{G}$ & Vosges & region & $\mathrm{b}$ & yes \\
\hline Dry 21 & dry stave & G & Vosges & region & $\mathrm{d}$ & yes \\
\hline Dry 22 & dry stave & G & Vosges & region & $\mathrm{b}$ & yes \\
\hline Dry 23 & dry stave & $\mathrm{G}$ & Vosges & region & $\mathrm{b}$ & yes \\
\hline Dry 24 & dry stave & I & Vosges & region & $\mathrm{c}$ & yes \\
\hline Dry 25 & dry stave & I & Vosges & region & $\mathrm{c}$ & yes \\
\hline Dry 26 & dry stave & I & Vosges & region & $\mathrm{c}$ & yes \\
\hline Dry 27 & dry stave & I & Vosges & region & $\mathrm{c}$ & yes \\
\hline Dry 28 & dry stave & $\mathrm{J}$ & Centre & region & $\mathrm{d}$ & yes \\
\hline Dry 29 & dry stave & $\mathrm{J}$ & Centre & region & $\mathrm{c}$ & yes \\
\hline Dry 30 & dry stave & $\mathrm{J}$ & Centre & region & $\mathrm{d}$ & yes \\
\hline Dry 31 & dry stave & $\mathrm{J}$ & Centre & region & $\mathrm{c}$ & yes \\
\hline Dry 32 & dry stave & $\mathrm{F}$ & Vezelay & sub-region & $\mathrm{d}$ & yes \\
\hline Dry 33 & dry stave & $\mathrm{F}$ & Vezelay & sub-region & $\mathrm{e}$ & yes \\
\hline Dry 34 & dry stave & $\mathrm{F}$ & Vezelay & sub-region & $\mathrm{d}$ & yes \\
\hline Dry 35 & dry stave & $\mathrm{F}$ & Vezelay & sub-region & - & - \\
\hline Dry 36 & dry stave & $\mathrm{F}$ & Vezelay & sub-region & $\mathrm{b}$ & yes \\
\hline Barrel 1 & barrel stave & $\mathrm{K}$ & Tronçais & stand & $\mathrm{c}$ & yes \\
\hline Barrel 2 & barrel stave & $\mathrm{K}$ & Tronçais & stand & $\mathrm{c}$ & yes \\
\hline Barrel 3 & barrel stave & $\mathrm{K}$ & Tronçais & stand & $\mathrm{a}$ & no \\
\hline Barrel 4 & barrel stave & $\mathrm{K}$ & Nevers & sub-region & $\mathrm{e}$ & yes \\
\hline Barrel 5 & barrel stave & $\mathrm{K}$ & Nevers & sub-region & c-e & yes \\
\hline Barrel 6 & barrel stave & $\mathrm{K}$ & Nevers & sub-region & d & yes \\
\hline Barrel 7 & barrel stave & $\mathrm{K}$ & Vosges & region & $\mathrm{a}$ & yes \\
\hline Barrel 8 & barrel stave & $\mathrm{K}$ & Vosges & region & $\mathrm{b}$ & yes \\
\hline Barrel 9 & barrel stave & $\mathrm{K}$ & Vosges & region & f-g-h & no \\
\hline Barrel 10 & barrel stave & $\mathrm{K}$ & Centre France & region & $a-c-e-f-g-h$ & yes \\
\hline Barrel 11 & barrel stave & $\mathrm{K}$ & Centre France & region & $\mathrm{e}^{-8}$ & yes \\
\hline Barrel 12 & barrel stave & $\mathrm{K}$ & Centre France & region & $\mathrm{e}$ & yes \\
\hline Barrel 13 & barrel stave & $\mathrm{K}$ & Allier & region & c-e & yes \\
\hline Barrel 14 & barrel stave & $\mathrm{K}$ & Allier & region & $\mathrm{c}$ & yes \\
\hline Barrel 15 & barrel stave & $\mathrm{K}$ & Allier & region & c-e & yes \\
\hline Barrel 16 & barrel stave & $\mathrm{K}$ & Slovakia & country & $\mathrm{f}$ & yes \\
\hline Barrel 17 & barrel stave & $\mathrm{K}$ & Slovakia & country & $\mathrm{f}$ & yes \\
\hline Barrel 18 & barrel stave & $\mathrm{K}$ & Slovakia & country & - & - \\
\hline Barrel 19 & barrel stave & $\mathrm{K}$ & Czech Republic & country & $a-c-e-f-g-h$ & yes \\
\hline Barrel 20 & barrel stave & $\mathrm{K}$ & Czech Republic & country & $f-g-h$ & yes \\
\hline Barrel 21 & barrel stave & $\mathrm{K}$ & Czech Republic & country & $a-f-g-h$ & yes \\
\hline Barrel 22 & barrel stave & $\mathrm{K}$ & Russia & country & - & - \\
\hline Barrel 23 & barrel stave & $\mathrm{K}$ & Russia & country & a-d-e-f-g-h-i & yes \\
\hline Barrel 24 & barrel stave & $\mathrm{K}$ & Russia & country & - & - \\
\hline Barrel 25 & barrel stave & $\mathrm{K}$ & Ukrainia & country & f-g-h & yes \\
\hline Barrel 26 & barrel stave & $\mathrm{K}$ & Ukrainia & country & $\mathrm{f}$ & yes \\
\hline Barrel 27 & barrel stave & $\mathrm{K}$ & Ukrainia & country & $\mathrm{b}$ & no \\
\hline Barrel 28 & barrel stave & $\mathrm{K}$ & United states & country & - & - \\
\hline Barrel 29 & barrel stave & $\mathrm{K}$ & United states & country & - & - \\
\hline Barrel 30 & barrel stave & $\mathrm{K}$ & United states & country & - & - \\
\hline
\end{tabular}


Table II. Primer pairs used for haplotype characterisation.

\begin{tabular}{|c|c|c|c|c|c|}
\hline Primer pair & $\begin{array}{c}\text { Primers } \\
\text { combination }\end{array}$ & Sequence $5^{\prime}-3^{\prime}$ & Primer & $\begin{array}{l}\text { Fragment length } \\
\text { (bp) }\end{array}$ & $\begin{array}{l}\text { Annealing } \\
\text { temperature }\end{array}$ \\
\hline \multirow[t]{2}{*}{ d1t1 } & I & GAGACCAGAAAGGGTAATGA & $\mathrm{d} 1$ & 350 & $49^{\circ} \mathrm{C}$ \\
\hline & & CTAAATAGCAACGCAAGAAA & $\mathrm{t} 1$ & & \\
\hline \multirow[t]{2}{*}{$\mathrm{d} 7 \mathrm{t} 7$} & I & GATCAATTCAATTAGGGTCG & d7 & 167 & $52{ }^{\circ} \mathrm{C}$ \\
\hline & & ATATCTTATGCACATGGTGG & $\mathrm{t} 7$ & & \\
\hline \multirow[t]{2}{*}{$\mathrm{t} 4 \mathrm{f} 4$} & I & AGCTGTTCTAACAAGTGGGG & $\mathrm{t} 4$ & 269 & $47^{\circ} \mathrm{C}$ \\
\hline & & GGACTCTATCTTTATTCTCG & $\mathrm{f} 4$ & & \\
\hline \multirow[t]{2}{*}{ dt $12 b$} & II & GAGACCAGAAAGGGTAATGAA & $\mathrm{dt} 12 \mathrm{bu}$ & 175 & $50^{\circ} \mathrm{C}$ \\
\hline & & TTTCAATAACTTGTTGATCC & $\mathrm{dt} 12 \mathrm{bl}$ & & \\
\hline \multirow[t]{2}{*}{$\mathrm{dt} 72$} & II & GCGTTGCTATTTAGTAAATCC & $\mathrm{dt} 72 \mathrm{u}$ & 113 & $50^{\circ} \mathrm{C}$ \\
\hline & & GTGGACCATTCAGGAACGAGA & $\mathrm{dt} 721$ & & \\
\hline \multirow[t]{2}{*}{$\mathrm{tf} 42$} & II & TAATGACGACCCGAATCTTTA & $\mathrm{tf} 42 \mathrm{u}$ & 69 & $45^{\circ} \mathrm{C}$ \\
\hline & & ACAACAACTCTTTCGATTTTT & $\mathrm{tf} 421$ & & \\
\hline \multirow[t]{2}{*}{$\mu \mathrm{dt} 1$} & III & ATCTTACACTAAGCTCGGAA & $\mu \mathrm{dt} 1 \mathrm{u}$ & 87 & $48^{\circ} \mathrm{C}$ \\
\hline & & TTCAATAACTTGTTGATCCC & $\mu \mathrm{dt} 11$ & & \\
\hline \multirow[t]{2}{*}{$\mathrm{dt} 13$} & III & GAGACCAGAAAGGGTAATGAA & $\mathrm{dt} 13 \mathrm{u}$ & 53 & $50^{\circ} \mathrm{C}$ \\
\hline & & GAATCAATGAATGAAAGTGGA & dt131 & & \\
\hline \multirow[t]{2}{*}{ dt73 } & III & GCGTTGCTATTTAGTAAATCC & $\mathrm{dt} 73 \mathrm{u}$ & 69 & $45^{\circ} \mathrm{C}$ \\
\hline & & AGAGTAAACTAGTGATTATGG & $\mathrm{dt} 731$ & & \\
\hline \multirow[t]{2}{*}{ dt74 } & III & CCATAATCACTAGTTTACTCT & $\mathrm{dt} 74 \mathrm{u}$ & 65 & $45^{\circ} \mathrm{C}$ \\
\hline & & GTGGACCATTCAGGAACGAGA & $\mathrm{dt} 741$ & & \\
\hline \multirow[t]{2}{*}{ dt73b } & III & GCGTTGCTATTTAGTAAATCC & $\mathrm{dt} 73 \mathrm{bu}$ & 62 & $45^{\circ} \mathrm{C}$ \\
\hline & & ACTAGTGATTATGGGTAAACC & $\mathrm{dt} 73 \mathrm{bl}$ & & \\
\hline \multirow[t]{2}{*}{$\mathrm{dt} 74 \mathrm{~b}$} & III & TTTACTCTATACTCACTAGAG & $\mathrm{dt} 74 \mathrm{bu}$ & 52 & $45^{\circ} \mathrm{C}$ \\
\hline & & GTGGACCATTCAGGAACGAGA & $\mathrm{dt} 74 \mathrm{bl}$ & & \\
\hline
\end{tabular}

obtained from the barrel. The set of five chloroplast fragments, $\mathrm{dt} 13$ and $\mu \mathrm{dt} 1$ (instead of dt12b), dt73 and dt74 (instead of dt72) and tf 42 , each containing a single informative polymorphism, permits the same haplotype distinction but with the analysis of shorter fragments. The adaptation of genetic analyses to wood samples, including the necessity to amplify very small fragments for increasingly degraded wood, as well as the haplotype characterisation based on the combination of several PCR-RFLP analyses, are described in Deguilloux et al. [5]. Each amplification experiment included both DNA isolation controls and PCR controls. Seven of the cpDNA fragments were digested by restriction endonucleases: $\mathrm{d} 7 \mathrm{t} 7, \mathrm{dt} 72, \mathrm{dt} 73$ and $\mathrm{dt} 74$ fragments with MseI, d1t1, dt12b and dt13 with TaqI, whereas others were analysed directly after PCR. Digestion controls (digestion of DNA isolated from fresh buds) were included in the experiment to check the digestion and to compare wood DNA digestion products with those of known haplotypes. PCR and digestion products were checked on $8 \%$ polyacrylamide gel followed by ethidium bromide staining and visualised under UV light, whereas the chloroplast microsatellite $\mu \mathrm{dt} 1$ was resolved on a Li-Cor model 4000L automatic DNA sequencer.

\subsection{Test of conformity of origin}

The use of the statistical procedure developed by Deguilloux et al. [4] allowed us to test if the combination of haplotypes found in the 11 wood lots analysed in this study were conforming to a French origin. Each wood lot corresponded to all wood samples originating from one particular cooperage industry. Wood samples of known foreign origin were therefore included in some of these lots, purely for demonstration and illustration purposes. In particular, the last lot corre- sponded to the "international" barrel. We define a null hypothesis $\mathrm{H}_{\mathrm{o}}$ : the wood lot is in fact of French origin. The number of samples from the wood lot that have been typed is called N. For each haplotype $i$ identified in the tested lot, $\mathrm{p}(\mathrm{i})$ denotes its frequency in the region of alleged origin (France in our case). We state that: if $p(i)<0.05$, then $P(i)=1-[1-\mathrm{p}(\mathrm{i})]^{\mathrm{N}}$, if $p(i) \geq 0.05$, then $P(i)=1$.

The probability $P$ to observe the particular configuration of haplotypes in the sample is given by the product of all $P(i): P=\prod_{i} P(i)$. If $P<S$ we will reject the hypothesis $H_{\mathrm{o}}$ (and declare that the lot is non-conform) with a risk $\alpha \leq S$, whereas if $P \geq S$ we cannot reject the hypothesis of conformity. Although the procedure can help decide that a given lot is not conform (with an estimated risk that must be assumed), we can in no way decide that it is conform, since we are unable to evaluate the corresponding risk (i.e. the risk to declare that the lot is conform when it is in fact non-conform).

\section{RESULTS}

\subsection{DNA amplification - genotyping}

The design of specific primers, amplifying fragments of various lengths, allowed us to obtain good amplification success rates on most types of samples (Fig. 1). Relatively long fragments could be amplified on DNA isolated from fresh oak wood, with success rates ranging from 78 to $93 \%$, whereas shorter fragments were amplified on DNA isolated from dry 


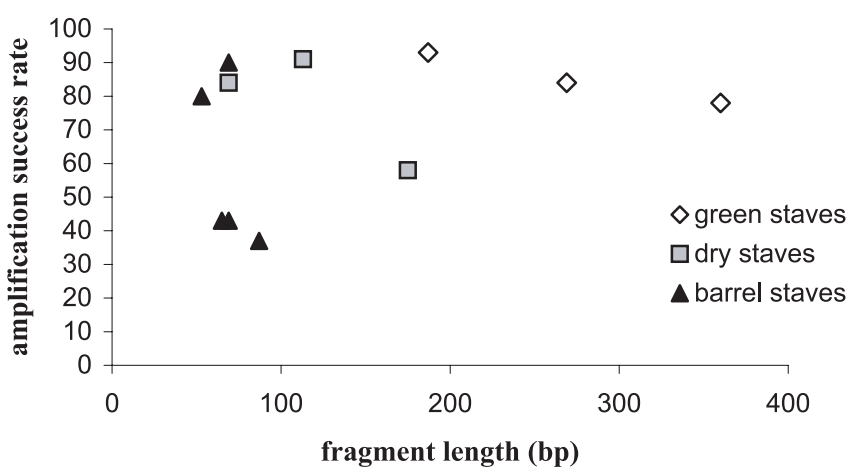

Figure 1. Amplification success rates of different chloroplast fragments over the 77 oak wood samples.

oak wood with success rates ranging from 58 to $91 \%$. Finally, no fragment longer than $90 \mathrm{bp}$ could be amplified on DNA isolated from barrel staves; with the shortest fragments the success rates ranged from 37 to $97 \%$. Amplification success was strongly dependent on the primer pairs used. The lowest success rates were for the fragments $\mu \mathrm{dt} 1$ (87 bp), dt73 (69 bp) and dt74 (65 bp) on barrel staves' DNA.

Whereas an important proportion of drying wood DNA could be genotyped with the PCR-RFLP combination I (i.e. with the longer fragments) (more than $86 \%$ of samples genotyped), shorter fragments were necessary to type dry wood DNA (Fig. 2). The best genotyping rates on every type of samples was obtained with combination III, with $96.2 \%$ of green staves and $95.6 \%$ of dry staves genotyped, compared to only $46.7 \%$ of the staves coming from the barrel.

In all cases, the same genotype was found with staves and logs corresponding to the same tree (Tab. I), confirming the relevance of the technique and the absence of contamination between samples or of contamination by external DNA. Moreover, all isolation and PCR controls remained negative.

\subsection{Haplotype conformity with putative provenance}

Over the 77 green woods genotyped, only nine staves and four logs, corresponding to five oak trees, had haplotypes that were not consistent with the provenance announced by the coopers (Tab. I). This involved four cases where the haplotypes revealed from the wood samples had not been identified in previous samples from the corresponding stand (provenances Tronçais and Jupilles) and one case where the haplotype characterised had never been detected in native oak material from France (haplotype $f$ characterised on staves Green dd'). Indeed, haplotype $\mathrm{f}$ is widely distributed in central and eastern Europe, from Italy and Germany to Russia, but is not considered native in France (this haplotype had been detected so far in only two other French populations over 743 populations analysed, at low frequency, and probably on introduced material [16]). Similarly, only one dry stave had a haplotype that was not consistent with its region of origin. Indeed, this sample (Dry 5) had haplotype h, never found so far in Alsace but widespread in Europe, especially from Hungary to Russia.

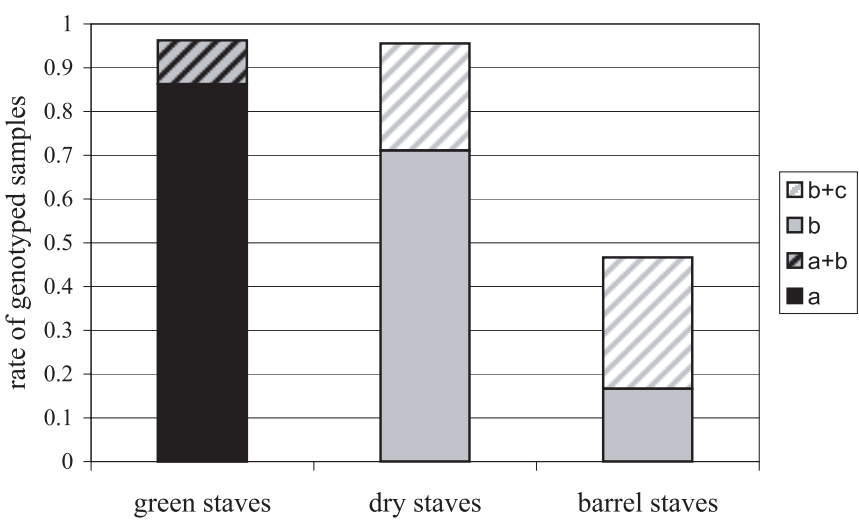

Figure 2. Proportion of green, dry and barrel staves genotyped with the PCR-RFLP combinations I, II or III.

For the barrel, three staves, among the 14 that could be genotyped, were not consistent with the origin of the wood lot used. These analyses were repeated subsequently for these three staves and the typing was fully confirmed. In one case (stave Barrel 3), the haplotype characterised is not known from the stand from where the wood used to make the barrel was thought to originate, whereas in the second case (stave Barrel 9), the sample was only partly genotyped, but all possible haplotypes (f, $g$ or $h$ ) have never been detected so far from Alsace, but are widespread in the central and eastern part of the continent. In the last case, a stave (Barrel 27) considered to originate from Ukrainia had a western European haplotype (haplotype b, which has never been found that far eastwards [16]).

\subsection{Test of conformity of origin}

Over the 11 wood lots considered, four were declared nonconsistent with a French origin at the 5\% threshold and two at the $1 \%$ threshold (Tab. III). This included lots from which characterised haplotypes are nearly absent from France (see last paragraph) as well as the barrel itself (made-up of several provenances, including some non-French ones).

\section{DISCUSSION}

The genetic methods designed for haplotype characterisation [5] proved to be relevant for woods used in the French barrel industry. Primer efficiency was very heterogeneous. In particular, it was clear that the shorter fragments did not always yield the best amplification rates, even though size played a large role. Indeed, the regions flanking informative polymorphisms are not always well suited for primer design, as in the case of fragment dt73, for which the formation of a dimer resulted in low amplification rates. In such cases, shifting primer position by a few nucleotides may make a large difference, as shown in Deguilloux et al. [5]. Nevertheless, it is now possible to characterise chloroplast haplotypes with a high success rate (over 95\%) on both green and dry oak staves, using those primers amplifying the shortest DNA fragments. 
Table III. Results of test of conformity on cooperage wood lots (c for conformity and nc for non conformity).

\begin{tabular}{|c|c|c|c|c|c|c|c|c|c|c|c|c|c|}
\hline \multirow{2}{*}{ Lot } & \multirow{2}{*}{ Size } & \multicolumn{9}{|c|}{ Haplotypes } & \multirow{2}{*}{$P$} & \multirow{2}{*}{$\begin{array}{c}\text { Threshold } \\
5 \%\end{array}$} & \multirow{2}{*}{$\begin{array}{c}\text { Threshold } \\
1 \%\end{array}$} \\
\hline & & $\mathrm{a}$ & $\mathrm{b}$ & $\mathrm{c}$ & $\mathrm{d}$ & $\mathrm{e}$ & $\mathrm{f}$ & $\mathrm{g}$ & $\mathrm{h}$ & $\mathrm{i}$ & & & \\
\hline A & 36 & 3 & 27 & 4 & 1 & 1 & - & - & - & - & 0.385 & $\mathrm{c}$ & $\mathrm{c}$ \\
\hline B & 26 & - & 22 & 2 & 3 & - & - & - & - & - & 1 & $\mathrm{c}$ & $\mathrm{c}$ \\
\hline $\mathrm{C}$ & 6 & 2 & 2 & 1 & - & - & - & - & 1 & - & 0.002 & $\mathrm{nc}$ & $\mathrm{nc}$ \\
\hline $\mathrm{D}$ & 5 & - & - & 4 & - & - & - & - & 1 & - & 0.021 & $\mathrm{nc}$ & $\mathrm{c}$ \\
\hline $\mathrm{E}$ & 3 & 1 & - & 1 & 1 & - & - & - & - & - & 0.039 & $\mathrm{nc}$ & $\mathrm{c}$ \\
\hline $\mathrm{F}$ & 5 & - & 2 & 2 & - & 1 & - & - & - & - & 1 & $\mathrm{c}$ & $\mathrm{c}$ \\
\hline $\mathrm{G}$ & 4 & - & 3 & - & 1 & - & - & - & - & - & 1 & $\mathrm{c}$ & $\mathrm{c}$ \\
\hline $\mathrm{H}$ & 4 & - & - & 4 & - & - & - & - & - & - & 1 & $\mathrm{c}$ & $\mathrm{c}$ \\
\hline I & 4 & - & - & 4 & - & - & - & - & - & - & 1 & $\mathrm{c}$ & $\mathrm{c}$ \\
\hline $\mathrm{J}$ & 4 & - & - & 2 & 2 & - & - & - & - & - & 1 & $\mathrm{c}$ & $\mathrm{c}$ \\
\hline $\mathrm{K}$ & 15 & 3 & 2 & 3 & 1 & 3 & 3 & - & - & - & 0.002 & $\mathrm{nc}$ & $\mathrm{nc}$ \\
\hline
\end{tabular}

Better amplification success rates and longer fragments could be obtained for green staves, compared to dry or toasted ones: clearly, the various treatments necessary to obtain the barrels alter the quality of the DNA present in the wood. In the cooperage industry, the traditional method to dry staves consists in storing the wood outdoors, generally up to two years. The wood humidity goes down from $60-75 \%$ to $12-18 \%$. Because this is a slow process, the risk of appearance of fissures in the staves is reduced [19]. As a consequence, however, the wood remains humid and exposed to oxygen and UV rays for a very long period, three factors that are known to affect DNA conservation [12]. Fortunately, although humidity favours the development of the microflora on the surfaces of the staves, fungi are reported to colonize only superficial layers of the wood [3, 20], which should restrict DNA degradation. On the other hand, during drying, oak wood undergoes a slow chemical and biochemical transformation, leading to the concentration of wood extractives [1]. A strong decline in the level of water-soluble ellagitanins has been reported in oaks staves, as part of these compounds is leached out by rainwater and another part is transformed by hydrolysis or oxidation into insoluble polymers [6]. Consequently, the open-air drying process results not only in highly degraded DNA but also in the apparition of molecules inhibiting genetic analyses (mainly PCR). Finally, during barrel making, the central parts of the staves are heated so that they can be shaped without splitting, and can release interesting extractible compounds in spirits. The heating of wood at a temperature up to $180{ }^{\circ} \mathrm{C}$ must further degrade DNA remains, leading to poorer haplotype determination rate on toasted staves compared to dried staves.

Despite these degradations of the DNA in oak staves, the adaptation of genetic analyses allowed us to determine the chloroplast haplotype in most cases. A reliable procedure can now be proposed to coopers, which permits to check the genetic conformity of oak wood with the announced provenance. Indeed, the comparison of chloroplast haplotypes identified on dry staves with that from fresh material directly sampled in the region of origin (by relying on previous studies of chloroplast DNA variability in Europe [16]) or in the stand itself, can be performed. The procedure could be improved by a more complete typing of cpDNA haplotypes. Indeed, the typing is still incomplete for haplotypes absent or very rare in France. The detection of new polymorphisms permitting to distinguish exotic haplotypes may be particularly worthwhile, as it would allow excluding more readily a given provenance. In any case, if precise information is provided on wood origin, and the area where the logs should have been collected is more precisely circumscribed, cases of non-conformity will be easier to identify with the genetic test. This is reflected in this study, where cases of nonconformity often included samples claimed to originate from specific stands. In these cases, only one or two haplotypes are expected, providing a higher exclusion power (by comparison, up to four or five different haplotypes can be expected from a given French "region"). However, according to our experience, wood provenance information is often vague, from the region to the country level. Additionally, there are also problems with provenance denomination, as the names used by coopers to identify regions often do not correspond to administrative ones.

Genetic analyses allowed us to check the genetic conformity between oak wood and claimed geographic origin, and can now be used in the barrel industry. Our first results proved the existence of unlabeled woods from eastern Europe and sold as French woods. Furthermore, wood suppliers appear to use the names of famous forests (such as Tronçais or Jupilles) in an improper way (for wood originating from other forests, regions, or countries). Our procedure could be used to protect the barrel industry from those abuses.

Provided precautions are taken against DNA contaminations during all analyses, haplotype determination on oak staves is possible. The procedure proposed should include the analysis of at least eight staves collected in the wood stock of a cooperage (staves collected during the drying process rather than toasted ones). This number (eight samples) allows the rejection of French origin from $4.6 \%$ of simulated French lots at the $5 \%$ threshold (i.e. the observed type I error was in good agreement with the predicted one, see [5]). The results (determination of the haplotype of each of these eight samples) can then be compared with the genetic composition of the announced provenance using the conformity test proposed. When the outcome is non-conformity with a French origin, a second analysis should be made to confirm this result. Finally, the result should be interpreted in the context of a more thorough 
investigation, as investigation is best viewed as a puzzle of indissociable elements.

Acknowledgements: We are grateful to the Demptos cooperage and Sogibois companies for providing numerous staves, to NadaliéLudonnaise cooperage for providing the "international" barrel and numerous dry staves, as well to Billon, Damy, Dargaud-Jaegle, François, Remond, Villard and Vicard cooperages for providing dry staves. We also thank F. Lagane and J.M. Louvet (INRA, Pierroton) who sawed the wood samples. This study has been carried out with financial support from the CTBA (Centre Technique du bois et de l'Ameublement), the Fédération Française de Tonnellerie, the Ministère de l'Agriculture et de la Pêche (DERF), the Ministère de la Recherche, the Commission of the European Communities (FEOGA in PDZR Aquitaine program and FOSSILVA project (EVK2-199900015P) and INRA (Institut National de la Recherche Agronomique).

\section{REFERENCES}

[1] Bauch J., Hundt H.V., Weißmann G., Lange W., Kubel H., On the cause of yellow discolorations of oak heartwood (Quercus Sect. Robur) during drying, Holzforschung 45 (1991) 79-85.

[2] Bazin J.P., Comment et pourquoi le goût du fût devint le goût du vin? Revue des Oenologues 98 (2001) 5-6.

[3] Chatonnet P., Boidron J.N., Dubourdieu D., Microflora of oak cask wood and their development during seasoning and aging in the open air, J. Int. Sci. Vigne Vin 28 (1994) 185-201.

[4] Deguilloux M.-F., Pemonge M.H., Petit R.J., Novel perspectives in wood certification and forensics: dry wood as a source of DNA, Proc. R. Soc. Lond. Ser. B Biol. Sci. 269 (2002) 1039-104.

[5] Deguilloux M.-F., Pemonge M.H., Bertel L., Kremer A., Petit R.J., Checking the geographical origin of oak wood: molecular and statistical tools, Mol. Ecol. 12 (2003) 1629-1636.

[6] De Simon B.F., Cadahia E., Conde E., Garcia-Vallejo M.C., Evolution of phenolic compounds of spanish oak wood during natural seasoning, First results, J. Agric. Food Chem. 47 (1999) 1687 1694.

[7] Dumolin-Lapègue S., Petit R.J., Gielly L., Taberlet P., Amplification of DNA from ancient and modern oak wood, Mol. Ecol. 8 (1999) 2137-2140.

[8] English N.B., Betancourt J.L., Dean J.S., Quade J., Strontium isotopes reveal distant sources of architectural timber in Chao Canyon, New Mexico, Proc. Nat. Acad. Sci. USA 98 (2001) 11891-11896.
[9] Feuillat F., Keller R., Variability of oak wood (Quercus robur L., Quercus petraea Liebl.) anatomy relating to cask properties, Am. J. Enol. Vitic. 48 (1997) 502-508.

[10] Francis I.L., Sefton M.A., Williams P.J., A study by sensory descriptive analysis of the effect of oak origin, seasoning, and heating on the aromas of oak model wine extracts, Am. J. Enol. Vitic. 43 (1992) 23-30.

[11] Hoffman E., Ludke C., Scholze H., Stephanowitch H., Analytical investigation of tree rings by laser ablation ICP-MS, Fresenius' J. Anal. Chem. 350 (1994) 253-259.

[12]Lindhal T., Instability and decay of the primary structure of DNA, Nature 362 (1993) 709-715.

[13] Marco J., Artajona J., Larrechi M.S., Rius F.X., Relationship between geographical origin and chemical composition of wood for oak barrels, Am. J. Enol. Vitic. 45 (1994) 192-200.

[14] Masson G., Guichard E., Fournier N., Puech J.L., Stereoisomers of Methyl- $\beta-\gamma$-octalactone, II, Contents in the wood of french (Quercus robur and Quercus petraea) and american (Quercus alba) oaks, Am. J. Enol. Vitic. 46 (1995) 424-428.

[15] Masson E., Baumes R., Moutounet M., Puech J.L., The effect of kiln-drying on the level of ellagitannins and volatile compounds of european oak (Quercus petraea Liebl.) stave wood, Am. J. Enol. Vitic. 51 (2000) 201-214.

[16] Petit R.J., Csaikl U.M., Bordacs S., Burg K., Coart E., Cottrell J., Van Dam B., Deans J.D., Dumolin-Lapègue S., Fineschi S., Finkeldey R., Gillies A., Glaz I., Goicoechea P.G., Jensen J.S., König A.O., Lowe A.J., Madsen S.F., Matyas G., Munro R.C., Olalde M., Pemonge M.H., Popescu F., Slade D., Tabbener H., Taurchini D., de Vries S.G.M., Ziegenhagen B., Kremer A., Chloroplast DNA variation in European white oaks phylogeography and patterns of diversity based on data from over 2600 populations, For. Ecol. Manage. 156 (2002) 5-26

[17] Sarni F., Moutounet M., Puech J.L., Rabier P., Effect of heat treatment of oak wood extractable compounds, Holzforschung 44 (1990) 461-466.

[18] Sauvageot F., Tessier C., Feuillat F., Variabilité (espèce, forêt, arbre, largeur de cernes et âge) de l'odeur du chêne français de tonnellerie (Quercus robur L., Quercus petraea Liebl.) étudiée par flairage de copeaux, Ann. For. Sci. 59 (2002) 171-184.

[19] Vivas N., Glories Y., Doneche B., Thoughts on natural drying of oak wood for barrel production, Rev. For. Fr. 48 (1996) 348-352.

[20] Vivas N., Amrani-Joutei K., Glories Y., Doneche B., Brechenmacher C., Développement de microorganismes dans le bois de coeur de chêne (Quercus petraea Liebl.) au cours du séchage naturel à l'air libre, Ann. Sci. For. 54 (1997) 563-571. 